\title{
Influence of La Doping on Magnetic and Optical Properties of Bismuth Ferrite Nanofibers
}

\author{
Ziang Zhang, ${ }^{1}$ Haiyang Liu,, ${ }^{1}$ Yuanhua Lin, ${ }^{1}$ Yan Wei, ${ }^{2}$ Ce-Wen Nan, ${ }^{1}$ and Xuliang Deng ${ }^{2}$ \\ ${ }^{1}$ State Key Laboratory of New Ceramics and Fine Processing, and Department of Materials Science and Engineering, \\ Tsinghua University, Beijing 100084, China \\ ${ }^{2}$ Department of Geriatric Dentistry, School and Hospital of Stomatology, Peking University, Beijing 100081, China
}

Correspondence should be addressed to Yuanhua Lin, linyh@mail.tsinghua.edu.cn and Yan Wei, kqdxl@mail.bjmu.edu.cn

Received 8 December 2011; Revised 1 March 2012; Accepted 2 March 2012

Academic Editor: Tong Lin

Copyright () 2012 Ziang Zhang et al. This is an open access article distributed under the Creative Commons Attribution License, which permits unrestricted use, distribution, and reproduction in any medium, provided the original work is properly cited.

The influence of La doping on the crystal structure, ferromagnetic, and optical properties of BFO nanofibers was investigated. $\mathrm{Bi}_{1-x} \mathrm{La}_{x} \mathrm{FeO}_{3}$ ultrafine nanofibers were synthesized by the electrospinning method. The surface morphology and crystal structure of the as-spun and sintered fibers were not affected by the doping. The impurity phases of the BFO crystals were weakened with the increment of La concentration. The magnetization field curves showed that the magnetization weakened under low La doping proportion, but strengthened with the increase of the doped proportion. The magnetization curves also showed continuous strong enhancement of ferromagnetic behavior. The results of UV-vis and photoabsorption testing revealed little influence of La doping on the optical property.

\section{Introduction}

Multiferroicity is a property that enables a material to display both ferroelectric and ferromagnetic properties. Due to its great potential in applications such as spintronics, memory, and data-storage media, multiferroicity has incited great research interest [1-4]. According to the phase-structure relationship, multiferroic materials can be divided into two groups: single phase and multiphase multiferroics, in which the electromagnetic coupling effect is greatly enhanced by the interaction between different phases composed of ferroelectric and ferromagnetic materials [5]. Single-phase multiferroics seldom exhibit both ferroelectric and ferromagnetic properties at room temperature. The one exception is $\mathrm{BiFeO}_{3}$ (BFO), which has a Curie temperature of $1103 \mathrm{~K}$ and a Neel temperature of $643 \mathrm{~K}$ and shows great merit in research and various applications $[2,6-8]$.

The structure of bulk BFO is perovskite crystal, with G-type ordering antiferromagnetic, in which the magnetic moment of $\mathrm{Fe}$ is ferromagnetic in $(001) \mathrm{h} /(111)$ c planes and antiferromagnetic in the neighboring two (001) $\mathrm{h} /(111)$ c planes [9]. A spin-modeling structure with a wavelength of $620 \pm 20 \AA$ can be observed, which eliminates the macroscopic magnetic moment of bulk BFO [10]. As a result, bulk BFO displays no macroscopic magnetic property. Suppression of the spin-modeling structure must be conducted in order to observe the ferromagnetic property of BFO. According to previous studies, the nanocrystalline effect can considerably enhance the ferromagnetism of $\mathrm{BFO}$ of low-dimensional form, where the mean particle size is reduced to below $100 \mathrm{~nm}[2,11-13]$. Another way which results in the cancellation of spin modeling is the doping of lanthanum (La) [14]. Besides the enhancement of ferromagnetism, there are also reports concerning the enhancement of photocatalytic activity brought by the synthesis of low-dimensional BFO, especially BFO nanofibers, whose fewer grain boundaries and interfaces effectively improve the photocatalytic property. Earlier studies have reported the structure, multiferroic properties of both Ladoped BFO bulk [15] and thin films [14, 16, 17] and ultrafine BFO nanofibers without doping [18]. Certain properties of La-doped BFO nanofibers, together with its optical properties, however, remain unexplored in the literature. 


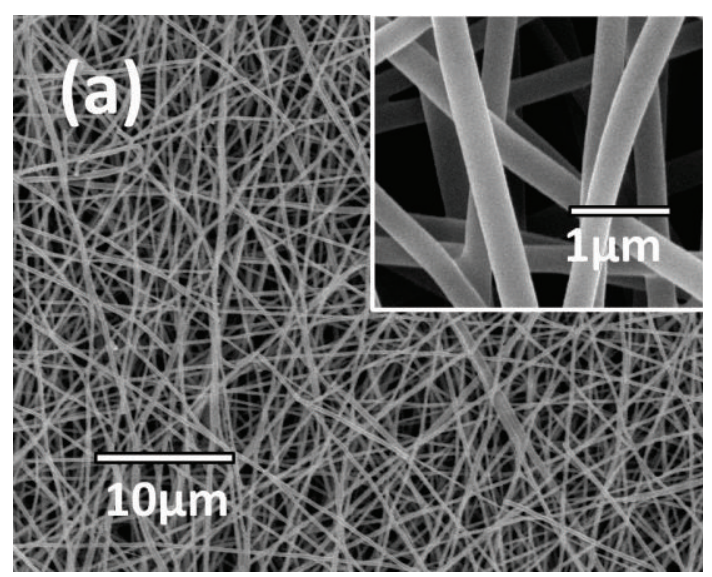

(a)

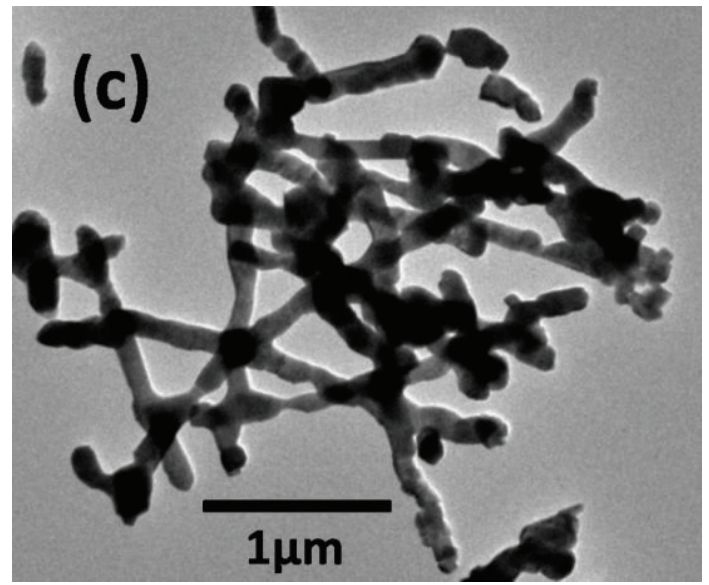

(c)

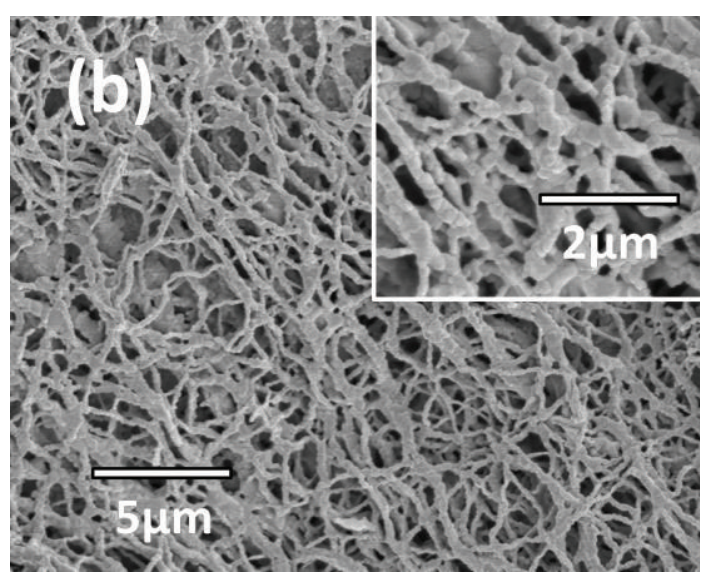

(b)

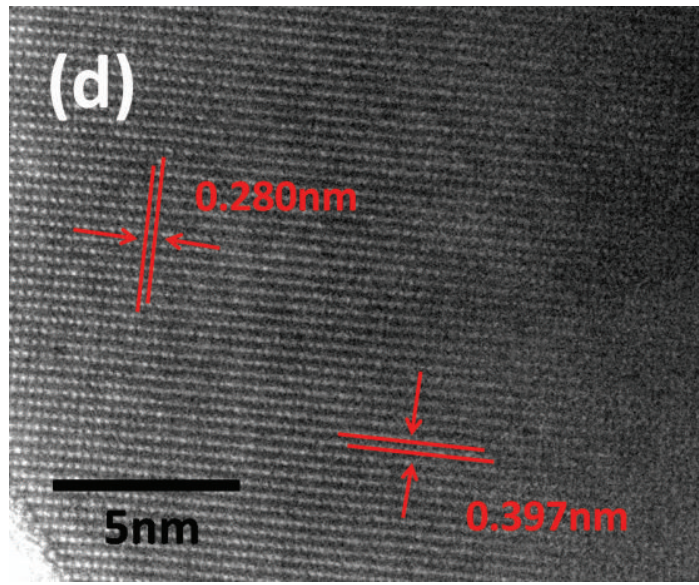

(d)

FIGURE 1: (a) SEM image of as-spun La-doped BFO nanofibers with 0.05 doping proportion, (b) SEM image, (c) TEM image, and (d) HR-TEM image of sintered La-doped BFO nanofibers with 0.05 doping proportion.

\section{Materials and Methods}

La-doped BFO $\left(\mathrm{Bi}_{1-x} \mathrm{La}_{x} \mathrm{FeO}_{3}\right)$ nanofibers with four scales of doping proportions $(x=0.0,0.05,0.10,0.15)$ are synthesized by sol-gel-based electrospinning. Bismuth nitrate $\left(\mathrm{Bi}\left(\mathrm{NO}_{3}\right)_{3} \cdot 5 \mathrm{H}_{2} \mathrm{O}\right)$ and iron nitrate $\left(\mathrm{Fe}\left(\mathrm{NO}_{3}\right)_{3} \cdot 9 \mathrm{H}_{2} \mathrm{O}\right)$ were dissolved in DMF (2-methoxyethanol, $\mathrm{C}_{3} \mathrm{H}_{8} \mathrm{O}_{2}$ ), with an initial ratio of $\mathrm{Bi}$ to $\mathrm{Fe}$ of $1.05(1-x): 1$, thus giving four sets of solutions. Lanthanum nitrate $\left(\mathrm{La}\left(\mathrm{NO}_{3}\right)_{3} \cdot 6 \mathrm{H}_{2} \mathrm{O}\right)$, also in stoichiometric proportion, was then added to the solution. After a period of aging, polyvinylpyrrolidone (PVP) was added to the solution, which was then stirred continuously, resulting in a homogeneous $0.3 \mathrm{M} \mathrm{Bi}_{1-x} \mathrm{La}_{x} \mathrm{FeO}_{3}$ solution. The solution was then loaded into a syringe with a stainless steel needle. High voltage was added to the needle to create a potential difference between the needle and the collector. The solution was then electrospun, with a feeding rate of $0.8 \mathrm{~mL} / \mathrm{h}$ and voltage of $13.5 \mathrm{kV}$. The fibers were collected on a Sn substrate. The as-spun nanofibers were then dried at $200^{\circ} \mathrm{C}$ for $1 \mathrm{~h}$, heated at $400^{\circ} \mathrm{C}$ for $1 \mathrm{~h}$, and then sintered at $600^{\circ} \mathrm{C}$ for $2 \mathrm{~h}$.

\section{Results and Discussion}

The scanning electron microscopy (SEM) images of both as-spun and sintered La-doped BFO fibers were taken, as shown in Figures 1(a) and 1(b). The diameter of the asspun nanofibers is approximately $300 \mathrm{~nm}$. The surface of the fibers is smooth, and they are uniform in shape. The sintered fibers are no longer uniform but rough, while the diameter of the fibers is reduced from approximately $300 \mathrm{~nm}$ to $100 \mathrm{~nm}$.

The crystalline structure of La-doped BFO ultrafine nanofibers is further examined by TEM and HRTEM, as shown in Figures $1(\mathrm{c})$ and $1(\mathrm{~d})$. It is observed from HRTEM images (Figure $1(\mathrm{~d})$ ) that the regular spacings of the observed lattice are 0.397 and $0.280 \mathrm{~nm}$, which are consistent with the (012) and (110) crystal planes of a rhombohedral La-doped BFO phase, respectively. In Figure 2, The EDX pattern of La-doped BFO nanofibers with different doping proportions are also included. From 0 to 0.15 loading content, the EDX pattern exhibits an increase in the La signal at $4.35 \mathrm{KeV}$, which indicates 


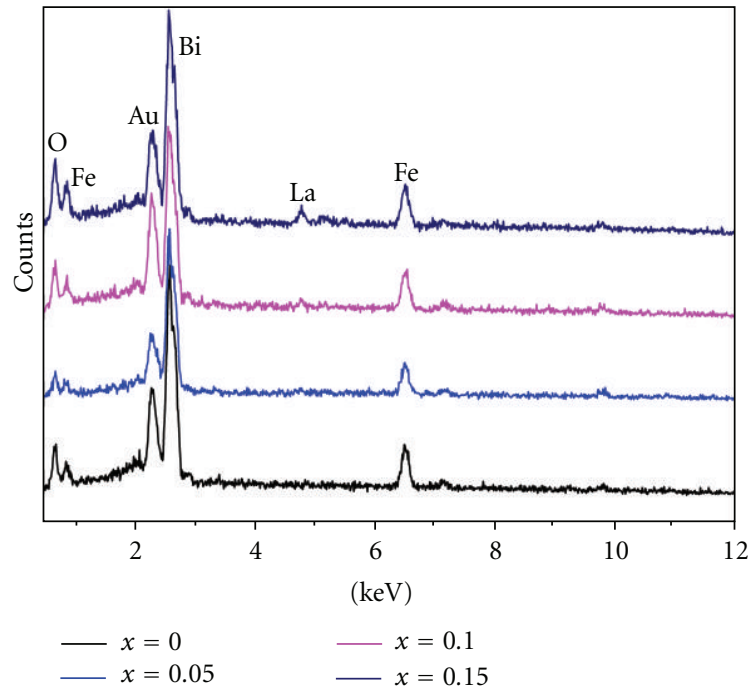

FIGURE 2: EDX pattern of La-doped BFO nanofibers with different doping proportions.

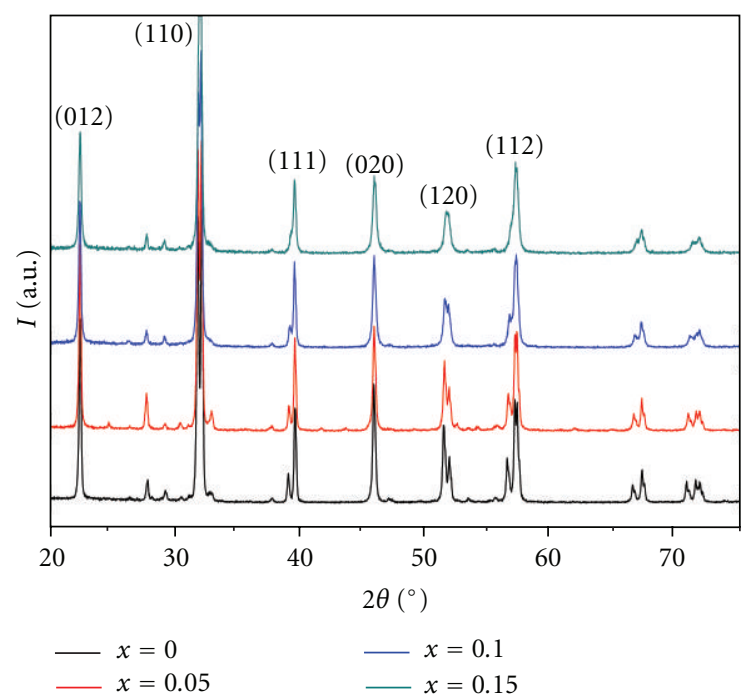

FIGURE 3: XRD spectra of La-doped BFO nanofibers with different doping proportions.

the formation of a La-doped BFO material. The percentages of atom numbers of La-doped $\mathrm{BFO}$ are shown in Table 2.

X-ray diffraction (XRD) was carried out to investigate the crystal structures of $\mathrm{BFO}$ with different $\mathrm{La}$ doping proportions. As shown in Figure 3, it can be observed that the rhombohedral perovskite structure of BFO contributes to most diffraction peaks. Ferromagnetic $\gamma-\mathrm{Fe}_{2} \mathrm{O}_{3}$, which often constitutes the main impurity phase of BFO, is not detected. The doping of La brings only minor change to the majority of the peaks, which indicates that the lattice constant of the perovskite crystal remains largely unchanged, as the difference between the ion diameters of $\mathrm{La}^{3+}(1.032 \AA)$ and $\mathrm{Bi}^{3+}(1.03 \AA)$ is trivial. It can also be observed that the
TABLE 1: Band gaps of sintered nanofibers under four La doping proportions.

\begin{tabular}{lc}
\hline La doping proportion $(x)$ & Band gap $(\mathrm{eV})$ \\
\hline 0.0 & 2.13 \\
0.05 & 2.13 \\
0.1 & 2.14 \\
0.15 & 2.10 \\
\hline
\end{tabular}

TABLE 2: The percentages of atom numbers of La-doped BFO nanofibers under four La doping proportions.

\begin{tabular}{lcccc}
\hline \multirow{2}{*}{ Element } & $x=$ & $x=$ & $x=$ & $x=$ \\
& $0.0(\%)$ & $0.05(\%)$ & $0.10(\%)$ & $0.15(\%)$ \\
\hline $\mathrm{O}$ & 61.3 & 59.7 & 59.1 & 58.0 \\
$\mathrm{Fe}$ & 20.0 & 20.4 & 21.0 & 21.7 \\
$\mathrm{La}$ & 0 & 1.1 & 2.0 & 2.8 \\
$\mathrm{Bi}$ & 18.7 & 18.8 & 17.9 & 17.5 \\
\hline
\end{tabular}

$28^{\circ}$ impurity peak is weakened with the doping of La. It is possible that the doping of La compensates the volatilization of $\mathrm{Bi}$, which otherwise leads to the existence of the $28^{\circ}$ peak during the preparation of the solution.

Considering the importance of the multiferroic property of $\mathrm{BFO}$, the ferromagnetic properties of La-doped BFO nanofibers are characterized by a vibrating sample magnetometer (VSM), as shown in Figure 4. From each of the four hysteresis loops corresponding to a La doping proportion, we can observe that the doping of La has obvious effect on the ferromagnetic properties of the nanofibers. With the increase of La concentration, the magnetization is at first $(x=0.05)$ weakened, as the doping of La leads to the reduction of $\mathrm{Fe}^{2+}$ and oxygen vacancies. The magnetization then increases $(x=0.10,0.15)$, as the spin-modeling structure is gradually suppressed by La doping. Saturation magnetization and coercive field, on the other hand, exhibit a continuous and evident increase with the increment of La doping.

Generally, the optical absorption performance of semiconductors is related to the electronic structure feature and their band gaps. The optical properties of samples were studied by measuring their UV-Vis diffuse reflectance absorption spectra. As shown in Figure 5, the absorption spectra of BFO nanofibers with different La doping proportions were measured. The absorption spectra show that BFO nanofibers can absorb considerable amounts of visible light, suggesting their potential applications as visible-light-driven photocatalysts. And compared with the BFO nanofibers with La doping, the pure BFO nanofibers show higher visiblelight absorption. The optical absorption coefficient near the band edge follows the equation $\alpha h v=A\left(h v-E_{g}\right)^{n / 2}$, where $\alpha, h, \nu, E_{g}$, and $A$ are absorption coefficient, Planck constant, light frequency, band gap, and a constant, respectively. Considering that $\mathrm{BFO}$ is a direct band gap material, the value of $n$ for $\mathrm{BiFeO}_{3}$ is 1.21 . The corresponding values of direct band gap of BFO nanofibers can be evaluated by extrapolating the linear portion of $(\alpha h \nu) 2$ versus $(h \nu)$; the 


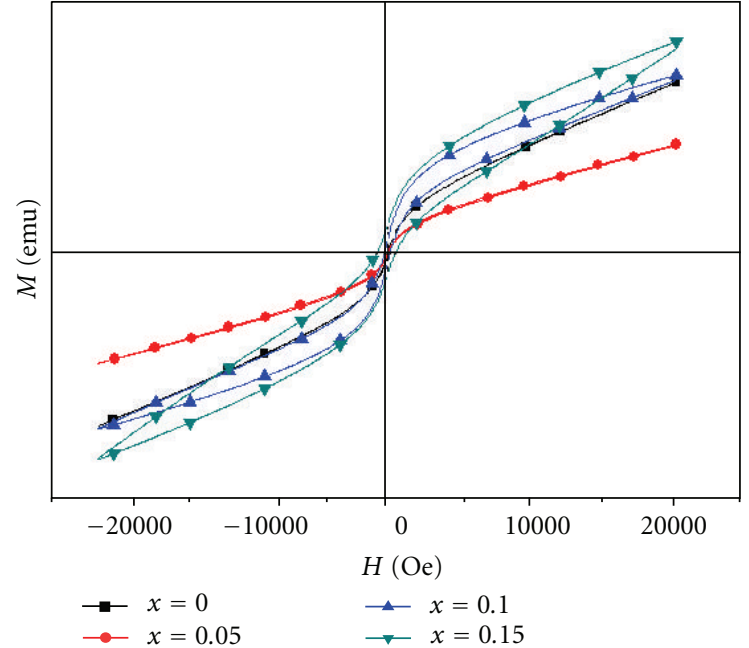

Figure 4: Magnetization field curves of La-doped BFO nanofibers with different doping proportions.

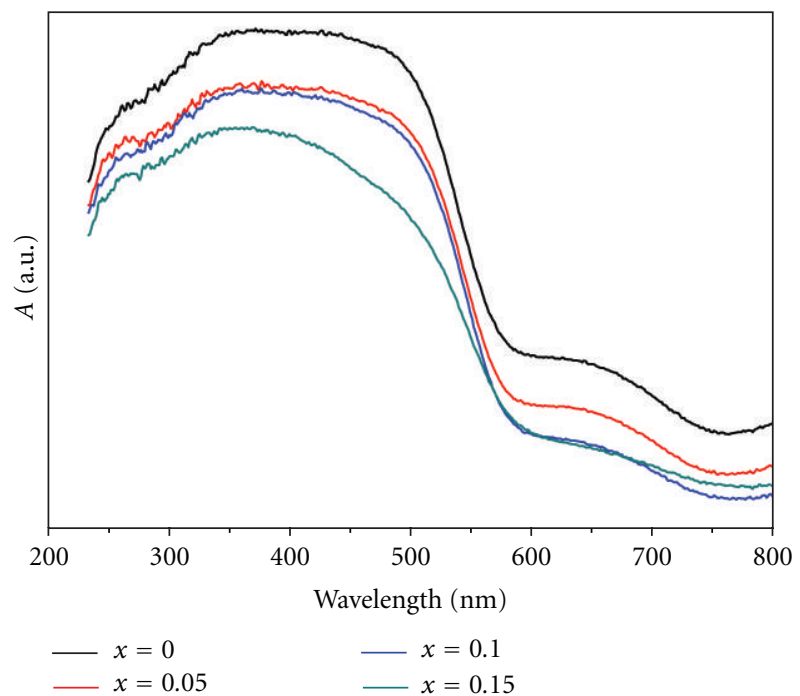

FIGURE 5: Ultraviolet and visible light absorbance of La-doped BFO nanofibers with different doping proportions.

results are shown in Table 1. The results show that La doping has little effect on the photoabsorbing property of BFO.

To further evaluate the photocatalytic properties of nanofibers, Congo red (CR) with a major absorption peak at $495 \mathrm{~nm}$ was chosen as a model organic pollutant. Figure 6 gives the concentration changes of CR at $495 \mathrm{~nm}$ by the nanofibers as a function of irradiation time under visible light during the degradation process. With La doping increased, $75 \%$ of the $\mathrm{CR}$ was decolorized after $3 \mathrm{~h}$, showing better photocatalytic activity than the pure BFO nanofibers. However, the enhanced activity of the photocatalysts does not increase further for more La doping. It can be seen that

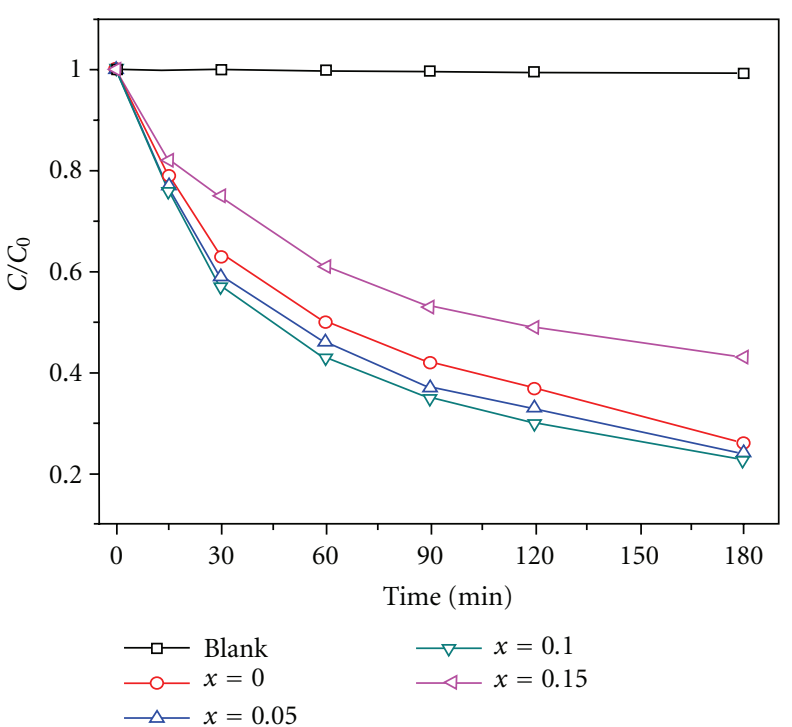

FIgURE 6: Photodegradation of CR in the presence of La-doped BFO nanofibers with different doping proportions.

the photodegradation rates of sample $(x=0.15)$ were lower than those of other samples.

\section{Conclusion}

In summary, the influence of La doping on the crystal structure, ferromagnetic, and optical properties of BFO nanofibers was investigated. $\mathrm{Bi}_{1-x} \mathrm{La}_{x} \mathrm{FeO}_{3}$ ultrafine nanofibers were synthesized by the electrospinning method. The surface morphology of as-spun and sintered fibers, as well as the crystal structure of sintered fibers, was not affected by the doping. The impurity phases of BFO crystals were weakened with the increment of La concentration. The magnetization field curves showed that the magnetization weakened under low La doping proportion, but strengthened with the increase of the doped proportion. The magnetization curves also showed continuous strong enhancement of ferromagnetic behavior. The results of UVVis and photoabsorption tests revealed little influence of $\mathrm{La}$ doping on the optical property.

\section{References}

[1] N. A. Hill, "Why are there so few magnetic ferroelectrics?" Journal of Physical Chemistry B, vol. 104, no. 29, pp. 66946709, 2000.

[2] J. Wang, J. B. Neaton, H. Zheng et al., "Epitaxial $\mathrm{BiFeO}_{3}$ multiferroic thin film heterostructures," Science, vol. 299, no. 5613, pp. 1719-1722, 2003.

[3] S. Y. Yang, F. Zavaliche, L. Mohaddes-Ardabili et al., "Metalorganic chemical vapor deposition of lead-free ferroelectric $\mathrm{BiFeO}_{3}$ films for memory applications," Applied Physics Letters, vol. 87, no. 10, Article ID 102903, pp. 1-3, 2005.

[4] M. Fiebig, T. Lottermoser, D. Frohlich, A. V. Golsev, and R. V. Pisarev, "Structural and electrical properties of $\mathrm{BiFeO}_{3}$ $\mathrm{Pb}(\mathrm{ZrTi}) \mathrm{O}_{3}$ composites," Nature, vol. 419, p. 819, 2002. 
[5] W. Eerenstein, N. D. Mathur, and J. F. Scott, "Multiferroic and magnetoelectric materials," Nature, vol. 442, no. 7104, pp. 759-765, 2006.

[6] T. Zhao, A. Scholl, F. Zavaliche et al., "Electrical control of antiferromagnetic domains in multiferroic $\mathrm{BiFeO} 3$ films at room temperature," Nature Materials, vol. 5, no. 10, pp. 823829, 2006.

[7] Y. H. Chu, L. W. Martin, M. B. Holcomb et al., "Electricfield control of local ferromagnetism using a magnetoelectric multiferroic," Nature Materials, vol. 7, no. 6, pp. 478-482, 2008.

[8] S. Lee, W. Ratcliff, S. W. Cheong, and V. Kiryukhin, "Electric

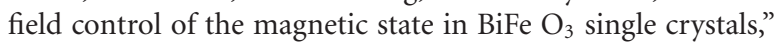
Applied Physics Letters, vol. 92, no. 19, Article ID 192906, 2008.

[9] C. Michel, J. M. Moreau, G. D. Achenbach, R. Gerson, and W. J. James, "The atomic structure of $\mathrm{BiFeO}_{3}$," Solid State Communications, vol. 7, no. 9, pp. 701-704, 1969.

[10] I. Sosnowska, T. P. Neumaier, and E. Steichele, "Spiral magnetic ordering in bismuth ferrite," Journal of Physics C, vol. 15, no. 23, pp. 4835-4846, 1982.

[11] T. J. Park, G. C. Papaefthymiou, A. J. Viescas, A. R. Moodenbaugh, and S. S. Wong, "Size-dependent magnetic properties of single-crystalline multiferroic $\mathrm{BiFeO}_{3}$ nanoparticles," Nano Letters, vol. 7, no. 3, pp. 766-772, 2007.

[12] F. Gao, Y. Yuan, K. F. Wang et al., "Preparation and photoabsorption characterization $\mathrm{BiFeO}_{3}$ nanowires," Applied Physics Letters, vol. 89, no. 10, Article ID 102506, 2006.

[13] S. Li, Y. H. Lin, B. P. Zhang, C. W. Nan, and Y. Wang, "Photocatalytic and magnetic behaviors observed in nanostructured $\mathrm{BiFeO}_{3}$ particles," Journal of Applied Physics, vol. 105, no. 5, Article ID 056105, 2009.

[14] Y. H. Lee, J. M. Wu, and C. H. Lai, "Influence of la doping in multiferroic properties of $\mathrm{BiFe}_{3}$ thin films," Applied Physics Letters, vol. 88, no. 4, Article ID 042903, pp. 1-3, 2006.

[15] Y. H. Lin, Q. Jiang, Y. Wang, C. W. Nan, L. Chen, and J. $\mathrm{Yu}$, "Enhancement of ferromagnetic properties in $\mathrm{BiFe} \mathrm{O}_{3}$ polycrystalline ceramic by la doping," Applied Physics Letters, vol. 90, no. 17, Article ID 172507, 2007.

[16] D. Lee, M. G. Kim, S. Ryu, H. M. Jang, and S. G. Lee, "Epitaxially grown La-modified $\mathrm{BiFeO}_{3}$ magnetoferroelectric thin films," Applied Physics Letters, vol. 86, no. 22, Article ID 222903, pp. 1-3, 2005.

[17] S. K. Singh and H. Ishiwara, "Doping effect of rare-earth ions on electrical properties of $\mathrm{BiFeO}_{3}$ thin films fabricated by chemical solution deposition," Japanese Journal of Applied Physics, vol. 45, no. 4 B, pp. 3194-3197, 2006.

[18] S. H. Xie, J. Y. Li, R. Proksch et al., "Nanocrystalline multiferroic $\mathrm{BiFeO}_{3}$ ultrafine fibers by sol-gel based electrospinning," Applied Physics Letters, vol. 93, no. 22, Article ID 222904, 2008. 

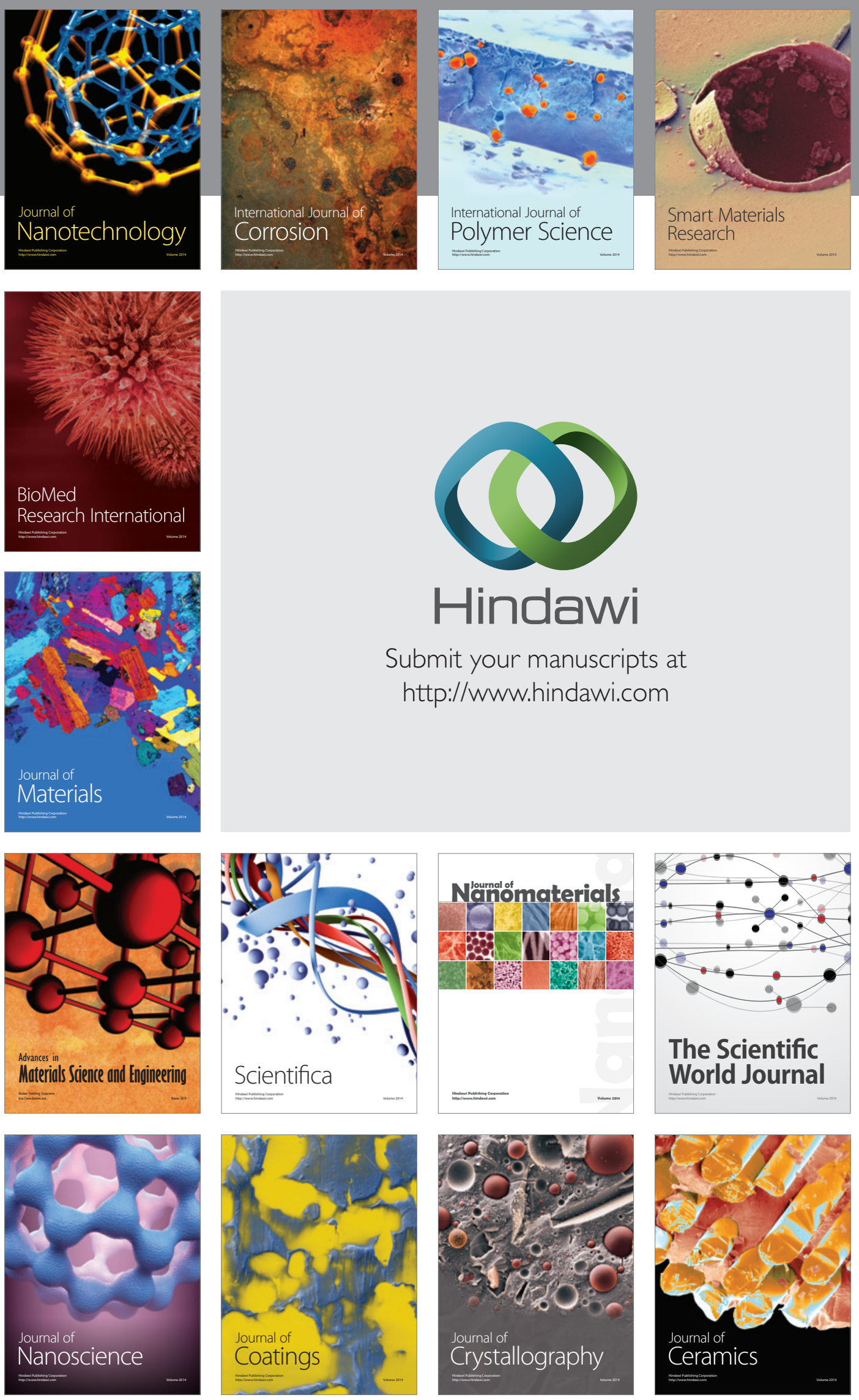

The Scientific World Journal

Submit your manuscripts at

http://www.hindawi.com

\section{World Journal}

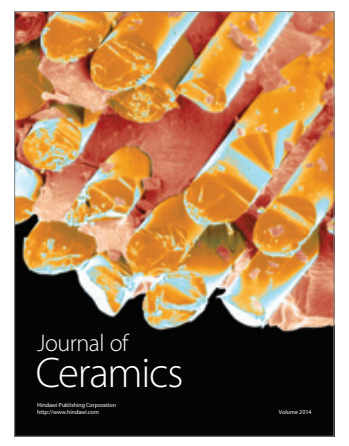

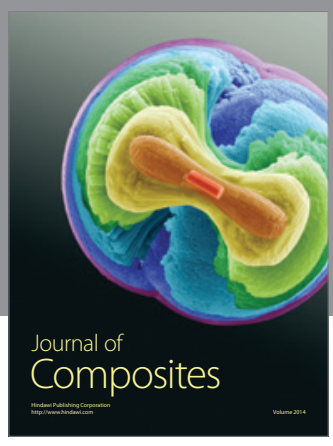
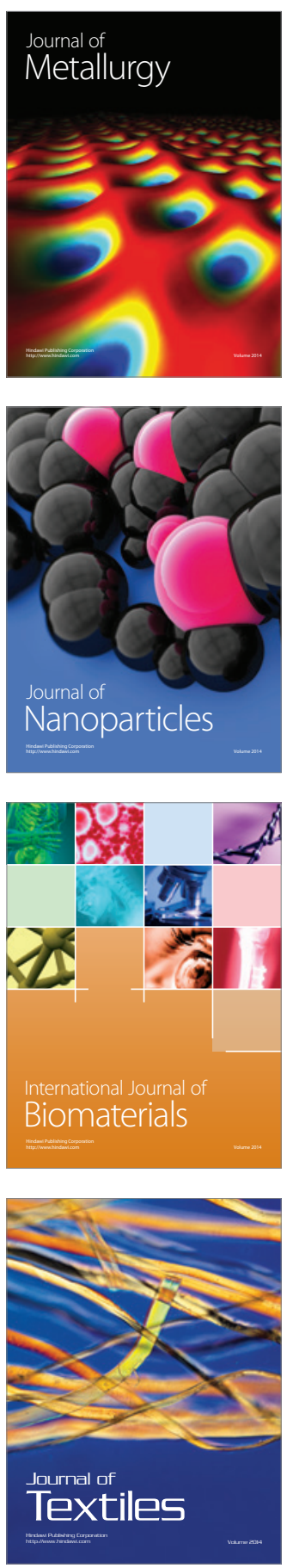